\title{
Systematics of Anopheles lanei Galvão and Amaral, a species of the subgenus Nyssorhynchus Blanchard (Diptera: Culicidae)
}

\author{
Luana Valente Senise, Maria Anice Mureb Sallum/+ \\ Departamento de Epidemiologia, Faculdade de Saúde Pública, Universidade de São Paulo, Av. Dr. Arnaldo 715 , \\ 01246-904 São Paulo, SP, Brasil
}

Anopheles (Nyssorhynchus) lanei Galvão and Amaral is here redescribed using morphological characteristics of adult, male and female, fourth instar larva and pupa. The larva, pupa, and male genitalia are illustrated. Diagnostic morphological characters of adults, male genitalia, fourth instar larva and pupa are provided to distinguish An. lanei from other species of the Argyritarsis section. Species distribution data are based on the published literature records and bionomics data are based on both literature records and field data.

Key words: Culicidae - Anopheles lanei - Nyssorhynchus - Systematics - Serra da Mantiqueira - Brazil

Anopheles Meigen includes 444 formally named species and 40 unnamed members of species complexes, divided into six subgenera (Harbach 2004). The subgenus Nyssorhynchus Blanchard includes some of the most important vectors of human malaria parasites in Central and South America (Forattini 2002).

Galvão and Amaral (1938) collected larvae and male and female adults of a species of Nyssorhynchus they described and named Anopheles lanei Galvão and Amaral. The type locality is Campos do Jordão (CJ) municipality, in Serra da Mantiqueira (SM), state of São Paulo, Brazil. The holotype and 22 paratypes were deposited in the Entomological Collection of Departmento de Parasitologia, Faculdade de Medicina - Universidade de São Paulo (FM-USP). Since its original description, few studies have been carried out on An. lanei. Pinto (1939) examined two paratypes, one male adult with associated genitalia slide and one female adult with associated larval skin, and confirmed that the anatomical characteristics defined by Galvão and Amaral (1938) could distinguish An. lanei from the remaining Anopheles species. Linthicum (1988) revised the systematics and internal classification of the Argyritarsis section, and included a redescription of all life stages of An. lanei and an illustration of male genitalia. Linthicum listed the individuals he used to redescribe $A n$. lanei, indicating that the specimens were from CJ; however, the illustration of the male genitalia was generated from a specimen collected in Estância (ET) municipality, state of Sergipe, Brazil. Morphologic evidence, in addition to ecological differences between CJ and ET, suggests that the specimens examined by Linthicum (1988) belonged to another species of Nyssorhynchus but not An. lanei. The objective of this study is to re-describe $A n$.

Financial support: FAPESP (05/53973-0) and CNPq (472485/ 2006-7; 135539/2005-6)

${ }^{+}$Corresponding author: masallum@usp.br

Received 25 July 2007

Accepted 13 December 2007 lanei using morphological characteristics of all life stages, except eggs, of specimens collected in the Pico do Itapeva, which is situated in between both Pindamonhagaba and CJ municipalities.

\section{MATERIALS AND METHODS}

Morphological characters of the adult male and female and the male genitalia were examined and measured. Pupal and larval chaetotaxy were examined, measured and counted for the descriptions. Nomenclature adopted for the descriptions of the adults male and female, larva and pupa follows Harbach and Knight (1980) and Forattini (1996), except for the wing spots and veins, which is that of Wilkerson and Peyton (1990) and Faran (1980). Measurements of the adults and immatures follow Belkin (1962). Morphological characters of seven male adults, 13 female adults, 20 larvae, 20 pupae and four male genitalia were measured. Immature stages mounted on microscope slides were measured using an ocular micrometer adapted to an optical microscope; the adults were measured with a digital ocular micrometer adapted to a stereomicroscope. Setae on Figs 1 and 2 do not represent the mode of branches showed in Tables I and II. Abbreviations for the life stages are: F, adult female; M, adult male; G, male genitalia; L, larva; Le, larval exuviae; Pe, pupal exuviae; E, egg. All the specimens are deposited in the Entomological Collection of Faculdade de Saúde Pública - Universidade de São Paulo (FSP-USP).

\section{TAXONOMIC TREATMENT}

Anopheles (Nyssorhynchus) lanei Galvão \& Amaral, 1938. Holotype female. Vila Emilio Ribas, Campos do Jordão, São Paulo, Brazil (FM-USP, 86-1, ACC n. 220, not found, possibly lost).

An. (Nyssorhynchus) lanei of Pinto 1939: 355, 387, 388 (paratype info.). Lane 1939: 25 (description info.). Galvão 1940: 474-475 (systematics and bionomics info.). Unti and Ramos 1942: 95-97, 101, 103 (distribution and bionomics info.). Unti 1943: 96-97, 99 (bionomics info.). Fonseca and Unti 1943: 45, 49-50 (infectivity info.). Russel et al. 1943: 37, 41, 46 (taxonomy, distribution and bionomics info.). Levi-Castillo 1949: $11,16,29,54-58,73,77,82,87,93,96,99,102$ (tax- 


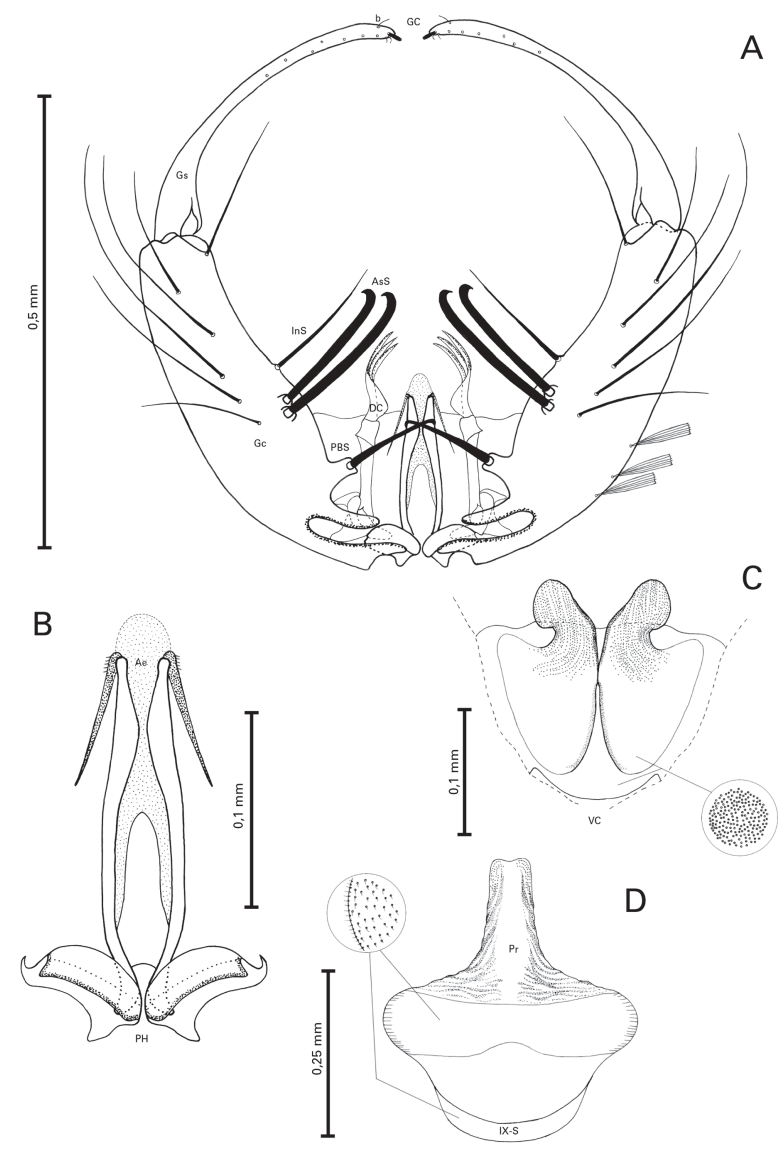

Fig. 1: An. lanei, male genitalia. A: dorsal view - gonocoxite (Gc); gonostylus (Gs); gonostylar claw (GC); setae b (b); internal seta (InS); accessory seta (AsS); parabasal seta (PBS); dorsal claspette (DC). B: phallosome (PH); aedeagus (Ae). C: ventral claspette (VC). D: proctiger (Pr), sternum IX (IX-S). Scales are in mm.

onomy, distribution, bionomics, $\left.\mathrm{L}^{*}, \mathrm{G}^{*}, \mathrm{~F}^{*}, \mathrm{E}^{*}\right)$. Lane 1953: 249-251 (taxonomy, $L^{*}, G^{*}, E^{*}$ ). Forattini 1962: $325,407,419-420,475,483,489$ (taxonomy, distribution, $\left.F^{*}, L^{*}, G^{*}\right)$.

Female. Head: integument dark brown; interocular space width 0.09-0.14 $\mathrm{mm}($ mean $=0.12 \mathrm{~mm})$, with 10 15 long pale setae and tuft of shorter, narrow pale scales; vertex with semi-erect spatulate pale scales; occiput with forked and long semi-erect pale scales medially and spatulate and broad dark scales laterally; ocular setae long, pale anteriorly and dark laterally, ocular scales broad and pale; gena, palpifer and clypeus bare, postgenal setae erected and dark. Antenna length $1.45-1.80 \mathrm{~mm}$ $($ mean $=1.60 \mathrm{~mm})$; pedicel and flagellum integument dark brown, pedicel with small, broad pale scales dorsally, flagellomere whorls with long dark setae, flagellomere 1 with a tuft of narrow, long, curved, spatulate pale scales, mostly on dorsal surface, flagellomeres 1-13 covered with minute whitish setae, flagellomere 1 1.05-1.30 $($ mean $=1.15)$ length of flagellomere 2 , antenna 0.60-0.70 $($ mean $=0.65)$ proboscis length. Maxillary palpus length $2.15-2.60 \mathrm{~mm}($ mean $=2.40 \mathrm{~mm})$; ra-

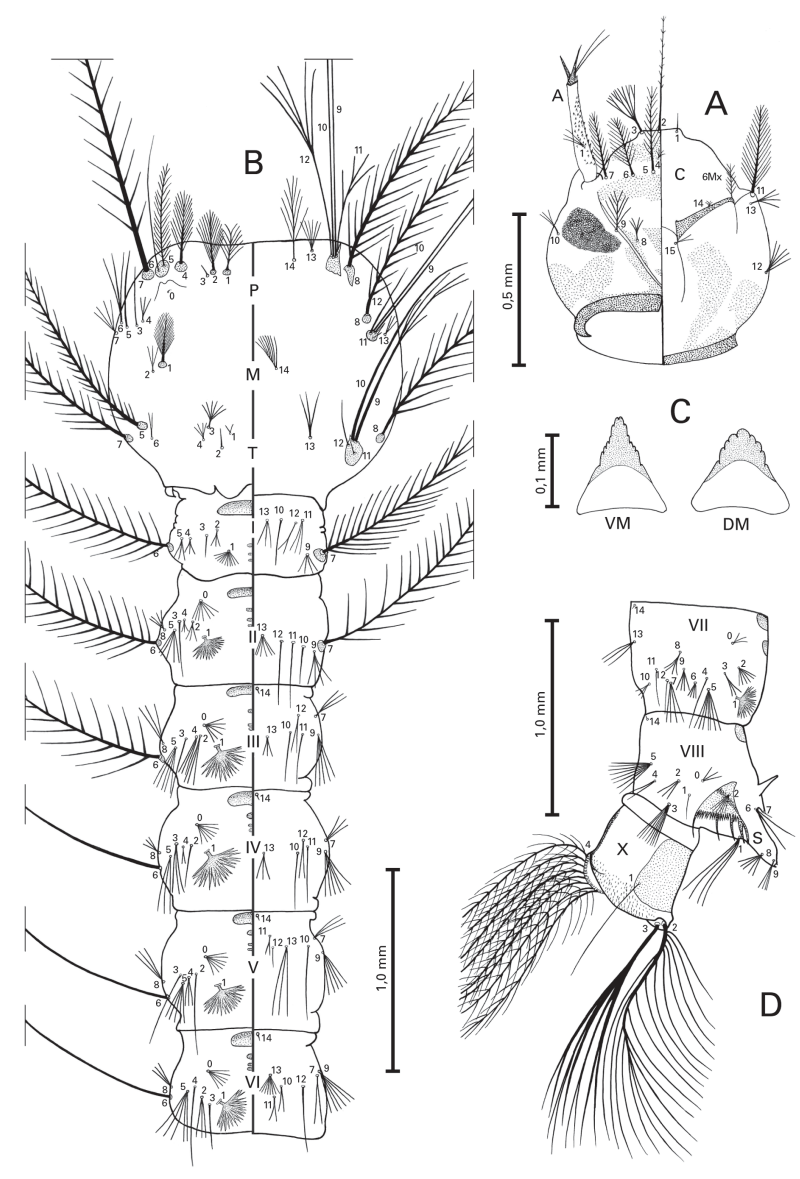

Fig. 2: An. lanei, fourth-instar larva. A: head - left side dorsal; right side ventral. B: thorax and abdominal segments I-VI - left side dorsal; right side ventral. C: dorsomentum (DM); ventromentum (VM). D: abdominal segments VII-X side view; spiracular apparatus (S). Scales are in $\mathrm{mm}$.

tio of length of palpomeres $1-5$ to total length of palpus, $1=0.05-0.07 ; 2=0.25-0.28 ; 3=0.34-0.38 ; 4=0.16-$ $0.19 ; 5=0.12-0.14 ;$ palpomere $20.70-0.85$ (mean $=$ 0.75 ) length of palpomere 3; palpomere 4 1.20-1.5 (mean $=1.30$ ) length of palpomere 5; maxillary palpus 1.20 1.35 (mean $=1.30)$ length of forefemur, 0.95-1.05 (mean $=1.00$ ) length of proboscis. Palpomere 1 and basal 0.3 of palpomere 2 with erect dark scales, distal 0.6 of palpomere 2 with semi-erect dark scales, palpomeres 3 with decumbent dark scales, joint of palpomeres 2-3 and apex of palpomere 3 with bands of pale scales dorsally, palpomere 4 with decumbent dark scales, palpomere 5 pale scaled, with long, whitish setae at apex. Proboscis length $2.05-2.70 \mathrm{~mm}$ (mean $=2.40 \mathrm{~mm}$ ), labial scales dark with golden sheen, labial basal setae long and dark. Thorax: scutal integument pruinose, with dark brown, non-pruinose, longitudinal stripes as follows: a median stripe along acrostichal area extending from anterior promontory to prescutellar area, a pair of broad, subdorsal stripes extending posteriorly from prescutal suture to scutellum, a pair of spots posterior to prescutal suture. Acrostichal, anterior dorsocentral, posterior 
TABLE I

Number of branches for setae of the larva of Anopheles (Nys.) lanei: range (mode) based on counts made on 20 to 24 setae

\begin{tabular}{|c|c|c|c|c|c|c|c|c|c|c|c|c|c|}
\hline \multirow{2}{*}{$\frac{\text { Seta }}{\mathrm{N}}$} & \multirow{2}{*}{$\frac{\text { Head }}{\mathrm{C}}$} & \multicolumn{3}{|c|}{ Thorax } & \multicolumn{9}{|c|}{ Abdominal segments } \\
\hline & & $\mathrm{P}$ & M & $\mathrm{T}$ & I & II & III & IV & $\mathrm{V}$ & VI & VII & VIII & $X$ \\
\hline 0 & 1 & 1 & - & - & - & $\begin{array}{c}7-12 \\
(9)\end{array}$ & $\begin{array}{c}7-12 \\
(9)\end{array}$ & $\begin{array}{c}1-11 \\
(9)\end{array}$ & $\begin{array}{c}2-9 \\
(6)\end{array}$ & $\begin{array}{c}3-7 \\
(5)\end{array}$ & $\begin{array}{c}2-4 \\
(3)\end{array}$ & $\begin{array}{c}2-4 \\
(3)\end{array}$ & - \\
\hline 1 & 1 & $\begin{array}{c}5-13 \\
(10)\end{array}$ & $\begin{array}{c}25-38 \\
(27)\end{array}$ & $\begin{array}{c}1-2 \\
(2)\end{array}$ & $\begin{array}{c}11-17 \\
(12)\end{array}$ & $\begin{array}{c}14-35 \\
(20)\end{array}$ & $\begin{array}{c}24-47 \\
(40)\end{array}$ & $\begin{array}{c}28-55 \\
(48)\end{array}$ & $\begin{array}{c}32-52 \\
(49)\end{array}$ & $\begin{array}{c}27-46 \\
(40)\end{array}$ & $\begin{array}{r}25-51 \\
(38)\end{array}$ & $\begin{array}{c}1-2 \\
(1)\end{array}$ & 1 \\
\hline 2 & 1 & $\begin{array}{c}12-24 \\
(18)\end{array}$ & $\begin{array}{c}1-5 \\
(3)\end{array}$ & 1 & $\begin{array}{c}3-4 \\
(3)\end{array}$ & $\begin{array}{c}4-7 \\
(5)\end{array}$ & $\begin{array}{c}3-4 \\
(3)\end{array}$ & $\begin{array}{c}1-2 \\
(1)\end{array}$ & $\begin{array}{c}1-2 \\
(1)\end{array}$ & $\begin{array}{l}3-6 \\
(4)\end{array}$ & $\begin{array}{c}4-8 \\
(6)\end{array}$ & $\begin{array}{c}4-6 \\
(4)\end{array}$ & $\begin{array}{c}13-22 \\
(18)\end{array}$ \\
\hline 3 & $\begin{array}{c}19-26 \\
(22)\end{array}$ & 1 & 1 & $\begin{array}{c}6-11 \\
(8)\end{array}$ & 1 & 1 & 1 & $\begin{array}{l}1-3 \\
(3)\end{array}$ & 1 & 1 & $\begin{array}{c}2-4 \\
(3)\end{array}$ & $\begin{array}{c}6-10 \\
(8)\end{array}$ & $\begin{array}{c}5-10 \\
(9)\end{array}$ \\
\hline 4 & $\begin{array}{l}2-7 \\
(4)\end{array}$ & $\begin{array}{c}13-27 \\
(19)\end{array}$ & $\begin{array}{l}2-5 \\
(4)\end{array}$ & $\begin{array}{l}3-5 \\
(4)\end{array}$ & $\begin{array}{l}2-4 \\
(3)\end{array}$ & $\begin{array}{l}3-5 \\
(4)\end{array}$ & $\begin{array}{l}2-4 \\
(3)\end{array}$ & $\begin{array}{l}2-4 \\
(3)\end{array}$ & $\begin{array}{l}1-3 \\
(3)\end{array}$ & 1 & 1 & $\begin{array}{c}1-2 \\
(2)\end{array}$ & $\begin{array}{l}8-9 \\
(8)^{a}\end{array}$ \\
\hline 5 & $\begin{array}{c}14-21 \\
(20)\end{array}$ & $\begin{array}{c}13-37 \\
(32)\end{array}$ & $\begin{array}{c}1-3 \\
(1)\end{array}$ & $\begin{array}{c}23-38 \\
(33)\end{array}$ & $\begin{array}{c}3-5 \\
(3)\end{array}$ & $\begin{array}{c}6-11 \\
(8)\end{array}$ & $\begin{array}{c}5-9 \\
(8)\end{array}$ & $\begin{array}{l}3-6 \\
(4)\end{array}$ & $\begin{array}{l}4-8 \\
(5)\end{array}$ & $\begin{array}{c}6-11 \\
(6)\end{array}$ & $\begin{array}{c}6-10 \\
(7)\end{array}$ & $\begin{array}{c}4-9 \\
(8)\end{array}$ & - \\
\hline 6 & $\begin{array}{c}16-23 \\
(21)\end{array}$ & 1 & $\begin{array}{c}3-6 \\
(3)\end{array}$ & $\begin{array}{c}1-2 \\
(2)\end{array}$ & $\begin{array}{c}21-33 \\
(26)\end{array}$ & $\begin{array}{c}25-33 \\
(28)\end{array}$ & $\begin{array}{c}14-24 \\
(18)\end{array}$ & 1 & 1 & 1 & $\begin{array}{c}4-8 \\
(5)\end{array}$ & $1-\mathrm{S}$ & $\begin{array}{c}6-9 \\
(6)\end{array}$ \\
\hline 7 & $\begin{array}{c}17-23 \\
(21)\end{array}$ & $\begin{array}{c}15-35 \\
(24)\end{array}$ & $\begin{array}{c}1-4 \\
(3)\end{array}$ & $\begin{array}{c}26-35 \\
(30)\end{array}$ & $\begin{array}{c}16-34 \\
(30)\end{array}$ & $\begin{array}{c}17-33 \\
(29)\end{array}$ & $\begin{array}{c}2-4 \\
(3)\end{array}$ & $\begin{array}{c}3-4 \\
(3)\end{array}$ & $\begin{array}{c}1-4 \\
(3)\end{array}$ & $\begin{array}{c}1-3 \\
(2)\end{array}$ & $\begin{array}{c}4-6 \\
(5)\end{array}$ & $2-\mathrm{S}$ & $\begin{array}{l}5-7 \\
(6)\end{array}$ \\
\hline 8 & $\begin{array}{c}3-6 \\
(5)\end{array}$ & $\begin{array}{c}15-34 \\
(29)\end{array}$ & $\begin{array}{c}15-34 \\
(27)\end{array}$ & $\begin{array}{c}19-41 \\
(30)\end{array}$ & - & $\begin{array}{c}2-6 \\
(4)\end{array}$ & $\begin{array}{c}3-6 \\
(4)\end{array}$ & $\begin{array}{c}2-5 \\
(3)\end{array}$ & $\begin{array}{c}2-5 \\
(3)\end{array}$ & $\begin{array}{c}3-4 \\
(3)\end{array}$ & $\begin{array}{c}3-6 \\
(5)\end{array}$ & $6-\mathrm{S}$ & $\begin{array}{l}1-6 \\
(2)\end{array}$ \\
\hline 9 & $\begin{array}{c}4-8 \\
(7)\end{array}$ & $\begin{array}{l}1-2 \\
(1)\end{array}$ & 1 & $\begin{array}{c}3-9 \\
(5)\end{array}$ & $\begin{array}{c}4-7 \\
(5)\end{array}$ & $\begin{array}{c}3-8 \\
(7)\end{array}$ & $\begin{array}{c}3-7 \\
(6)\end{array}$ & $\begin{array}{c}5-9 \\
(6)\end{array}$ & $\begin{array}{c}4-7 \\
(6)\end{array}$ & $\begin{array}{c}3-9 \\
(6)\end{array}$ & $\begin{array}{c}4-8 \\
(6)\end{array}$ & $7-\mathrm{S}$ & $\begin{array}{c}1-2 \\
(1)\end{array}$ \\
\hline 10 & $\begin{array}{c}2-4 \\
(3)\end{array}$ & 1 & $\begin{array}{c}1-2 \\
(1)\end{array}$ & 1 & 1 & 1 & $\begin{array}{c}1-2 \\
(1)\end{array}$ & $\begin{array}{c}1-2 \\
(1)\end{array}$ & 1 & $\begin{array}{c}2-3 \\
(2)\end{array}$ & $\begin{array}{c}2-4 \\
(3)\end{array}$ & $8-\mathrm{S}$ & $\begin{array}{c}4-7 \\
(6)\end{array}$ \\
\hline 11 & 30 & $\begin{array}{c}2-10 \\
(4)\end{array}$ & 1 & 1 & $\begin{array}{c}3-5 \\
(3)\end{array}$ & 1 & $\begin{array}{c}1-2 \\
(1)\end{array}$ & $\begin{array}{c}1-3 \\
(1)\end{array}$ & $\begin{array}{c}1-3 \\
(2)\end{array}$ & $\begin{array}{c}1-2 \\
(2)\end{array}$ & $\begin{array}{c}1-2 \\
(1)\end{array}$ & $9-\mathrm{S}$ & $\begin{array}{c}3-6 \\
(5)\end{array}$ \\
\hline 12 & $\begin{array}{l}3-7 \\
(5)\end{array}$ & $\begin{array}{c}2-9 \\
(5)\end{array}$ & 1 & $\begin{array}{c}1-2 \\
(1)\end{array}$ & $\begin{array}{l}1-3 \\
(2)\end{array}$ & 1 & $\begin{array}{l}1-3 \\
(2)\end{array}$ & $\begin{array}{l}1-3 \\
(2)\end{array}$ & $\begin{array}{c}1-3 \\
(1)\end{array}$ & 1 & $\begin{array}{c}1-2 \\
(1)\end{array}$ & - & - \\
\hline 13 & $\begin{array}{c}2-6 \\
(5)\end{array}$ & $\begin{array}{c}5-9 \\
(6)\end{array}$ & $\begin{array}{c}4-6 \\
(4)\end{array}$ & $\begin{array}{c}2-5 \\
(3)\end{array}$ & $\begin{array}{c}2-5 \\
(4)\end{array}$ & $\begin{array}{c}5-10 \\
(7)\end{array}$ & $\begin{array}{c}2-8 \\
(3)\end{array}$ & 3 & 3 & $\begin{array}{c}6-10 \\
(8)\end{array}$ & $\begin{array}{c}3-4 \\
(3)\end{array}$ & - & - \\
\hline 14 & $\mathrm{nc}$ & $\begin{array}{c}4-13 \\
(10)\end{array}$ & $\begin{array}{c}4-12 \\
(8)\end{array}$ & - & - & - & 1 & 1 & 1 & 1 & 1 & $\begin{array}{c}1-3 \\
(1)\end{array}$ & - \\
\hline 15 & $\begin{array}{c}1-5 \\
(3)\end{array}$ & - & - & - & - & - & - & - & - & - & - & - & - \\
\hline
\end{tabular}

a: number of pairs. nc: not counted; P: prothorax; M: mesothorax; T: metathorax.

dorsocentral, antealar, anterior scutal fossal, lateral scutal fossal, median scutal fossal, posterior medial scutal, posterior scutal fossal, prescutellar and supraalar setae strongly developed, long, dark brown; median anterior promontory setae long, whitish, silver; acrostichal, anterior dorsocentral, antealar, posterior dorsocentral, posterior scutal fossal, lateral scutal fossal, median scutal fossal, lateral prescutellar, median prescutellar and supraalar scales spatulate, white. Scutellum integument grayish, with a median dark spot, 13-16 long, dark setae, 6-11 shorter, whitish setae, white spatulate scales along posterior margin. Mesopostnotum bare, dark brown with few light brown areas. Pleural integument dark brown with pollinosity; metepisternum whitish; postprocoxal membrane, anepisternal cleft, lower anterior and medial mesokatepisternum, medial proepisternum and postpronotum, medial and anterior mesanepimeron and above prealar knob grayish. Antepronotum with 2-6 $($ mode $=4)$ ventral setae, 4-7 $($ mode $=6)$ dorsal setae, a tuft of dorsal dark scales. Setae on pleural areas as follow: postspiracular setae absent; reduced and few pale prespiracular; few, moderately developed upper proepisternal; few, moderately developed upper and lower mesokatepisternal, numerous, moderately developed upper mesepimeral and prealar; most pleural setae dark brown, prespiracular setae pale yellow. Scales on pleural areas as follow: few lower prealar, lower mesokatepisternal, upper mesepimeral scales. Basalare with few dark setae and patch of pale scales. Halter: integument yellowish, scabellum bare, pedicel with some 
TABLE II

Number of branches for setae of the pupa of Anopheles (Nys.) lanei: range, (mode) based on counts made on 20 to 24 setae

\begin{tabular}{|c|c|c|c|c|c|c|c|c|c|c|c|}
\hline \multirow{2}{*}{$\frac{\text { Seta }}{\mathrm{N}}$} & \multirow{2}{*}{$\begin{array}{c}\text { Cephalothorax } \\
\text { CT }\end{array}$} & \multicolumn{8}{|c|}{ Abdominal segments } & \multirow[b]{2}{*}{ IX } & \multirow{2}{*}{$\frac{\text { Padle }}{\mathrm{P}}$} \\
\hline & & I & II & III & IV & $\mathrm{V}$ & VI & VII & VIII & & \\
\hline 0 & - & - & $\begin{array}{c}3-7 \\
(4)\end{array}$ & $\begin{array}{l}2-5 \\
(4)\end{array}$ & $\begin{array}{l}3-5 \\
(4)\end{array}$ & $\begin{array}{r}2-5 \\
(3)\end{array}$ & $\begin{array}{c}2-4 \\
(3)\end{array}$ & $\begin{array}{r}2-4 \\
(3)\end{array}$ & $\begin{array}{l}1-2 \\
(1)\end{array}$ & - & - \\
\hline 1 & $\begin{array}{l}1-3 \\
(2)\end{array}$ & $\mathrm{nc}$ & $\begin{array}{c}3-8 \\
(6)\end{array}$ & $\begin{array}{l}2-6 \\
(5)\end{array}$ & $\begin{array}{l}1-2 \\
(1)\end{array}$ & 1 & $\begin{array}{c}1-2 \\
(1)\end{array}$ & $\begin{array}{c}1-2 \\
(1)\end{array}$ & - & $\begin{array}{c}2-6 \\
(3)\end{array}$ & 1 \\
\hline 2 & $\begin{array}{l}1-3 \\
(2)\end{array}$ & $\begin{array}{c}1-5 \\
(3)\end{array}$ & $\begin{array}{c}1-7 \\
(5)\end{array}$ & $\begin{array}{l}2-5 \\
(4)\end{array}$ & $\begin{array}{c}1-4 \\
(3)\end{array}$ & $\begin{array}{c}1-3 \\
(3)\end{array}$ & $\begin{array}{c}1-3 \\
(2)\end{array}$ & $\begin{array}{c}2-3 \\
(2)\end{array}$ & - & - & $\begin{array}{c}1 \\
(1)\end{array}$ \\
\hline 3 & $\begin{array}{l}2-3 \\
(3)\end{array}$ & $\begin{array}{c}1-3 \\
(1)\end{array}$ & $\begin{array}{l}1-3 \\
(1)\end{array}$ & $\begin{array}{c}1-2 \\
(1)\end{array}$ & $\begin{array}{l}1-5 \\
(2)\end{array}$ & $\begin{array}{l}1-4 \\
(3)\end{array}$ & $\begin{array}{c}1-4 \\
(2)\end{array}$ & $\begin{array}{c}2-4 \\
(2)\end{array}$ & - & - & - \\
\hline 4 & $\begin{array}{l}1-5 \\
(3)\end{array}$ & $\begin{array}{c}1-5 \\
(4)\end{array}$ & $\begin{array}{c}1-6 \\
(2)\end{array}$ & $\begin{array}{c}1-3 \\
(2)\end{array}$ & $\begin{array}{c}1-3 \\
(3)\end{array}$ & $\begin{array}{c}1-4 \\
(3)\end{array}$ & $\begin{array}{c}1-3 \\
(2)\end{array}$ & $\begin{array}{c}1-3 \\
(2)\end{array}$ & $\begin{array}{c}2-4 \\
(3)\end{array}$ & - & - \\
\hline 5 & $\begin{array}{r}2-5 \\
(4)\end{array}$ & $1-3(2)$ & $3-6(4)$ & $2-6(4)$ & 1 & 1 & 1 & 1 & - & - & - \\
\hline 6 & $\begin{array}{l}1-3 \\
(1)\end{array}$ & $\begin{array}{c}1-2 \\
(2)\end{array}$ & $\begin{array}{c}2-5 \\
(2)\end{array}$ & $\begin{array}{c}1-6 \\
(2)\end{array}$ & $\begin{array}{l}1-2 \\
(2)\end{array}$ & $\begin{array}{c}1-2 \\
(1)\end{array}$ & $\begin{array}{c}1-2 \\
(1)\end{array}$ & $\begin{array}{c}1-2 \\
(1)\end{array}$ & - & - & - \\
\hline 7 & $\begin{array}{l}2-3 \\
(3)\end{array}$ & $\begin{array}{l}2-5 \\
(3)\end{array}$ & $\begin{array}{c}2-6 \\
(4)\end{array}$ & $\begin{array}{l}1-5 \\
(2)\end{array}$ & $\begin{array}{c}1-4 \\
(3)\end{array}$ & $\begin{array}{c}2-4 \\
(3)\end{array}$ & $\begin{array}{c}1-2 \\
(1)\end{array}$ & $\begin{array}{c}1-2 \\
(1)\end{array}$ & - & - & - \\
\hline 8 & 1 & - & - & $\begin{array}{l}1-4 \\
(3)\end{array}$ & $\begin{array}{c}1-3 \\
(2)\end{array}$ & $\begin{array}{c}1-3 \\
(3)\end{array}$ & $\begin{array}{c}1-4 \\
(2)\end{array}$ & $\begin{array}{c}1-4 \\
(3)\end{array}$ & - & - & - \\
\hline 9 & $\begin{array}{c}1-3 \\
(2)\end{array}$ & $\begin{array}{c}1-2 \\
(1)\end{array}$ & 1 & 1 & 1 & 1 & 1 & 1 & 1 & - & - \\
\hline 10 & 1 & - & - & $\begin{array}{c}1-3 \\
(2)\end{array}$ & 1 & 1 & - & $\begin{array}{c}1-2 \\
(1)\end{array}$ & - & - & - \\
\hline 11 & $\begin{array}{c}2-3 \\
(3)\end{array}$ & - & - & 1 & 1 & 1 & 1 & $\begin{array}{c}1-3 \\
(2)\end{array}$ & - & - & - \\
\hline 12 & $\begin{array}{c}1-4 \\
(3)\end{array}$ & - & - & - & - & - & - & - & - & - & - \\
\hline 13 & - & - & - & - & - & - & - & - & - & - & - \\
\hline 14 & - & - & - & - & 1 & 1 & 1 & 1 & 1 & - & - \\
\hline
\end{tabular}

nc: not counted.

pale scales distally, capitellum proximal surface bare, dorsal and distal surfaces covered with pale yellow scales. Abdomen: integument dark brown, with grayish pollinosity, abdominal scales spatulate, broad spatulate on sterna; length $3.50-4.0 \mathrm{~mm}($ mean $=3.70 \mathrm{~mm})$; tergum I without scales, with sparse dark setae, short pale setae medially, terga II-VII with pearly scales medially, mainly posteriorly, with a pair of dark scale spots posteriorly, long dark brown setae sparse on surface, mostly laterally, posterolateral tufts of dark scales present on terga IV-VII, rarely on III, always absent on II; sternum I with sparse dark setae, sterna II-VII with sparse dark setae and pale scales medially. Segment VIII: tergum covered with golden yellow scales, sternum with dark scales medially and pale scales laterally, sparse dark setae on ventral and dorsal surfaces. Legs: integument and pattern of dark and pale scales and setae on coxae, trochanters, femora and tibiae similar to Argyritarsis Section (see Linthicum 1988 for details); foretarsomeres 1, 2 and 3 dark, with apical pale ring, foretarsomeres 4 and 5 en- tirely dark; anterior and posterior surfaces of midtarsomere 1 dark, with apical pale ring, a midlongitudinal pale stripe on ventral and dorsal surfaces; midtarsomeres 2 and 3 dark, with apical pale ring, midtarsomere 4 and 5 entirely dark, with some lighter dark scales at apex of midtarsomere 5; hindtarsomere 1 dark, with evident apical pale ring and midlongitudinal pale stripe on ventral and dorsal surfaces, hindtarsomere 2 dark-scaled at basal 0.30-0.52 (mean $=0.45)$, hindtarsomeres 3, 4 and 5 entirely white scaled. Wing: dark scales dark brown to black, pale scales white, length 3.70-4.70 $\mathrm{mm}($ mean $=4.5 \mathrm{~mm})$; basal dark, sector pale, accessory sector pale and preapical pale spots absent, humeral dark spot either absent or present, subcostal pale and prehumeral dark present, basal pale and prehumeral pale fused into a single basal pale spot, presector dark and sector dark fused into a single medial dark spot, preapical dark and apical dark fused into a single apical dark spot. Veins $S C, R_{1}$ and parts of $R_{S}$ and $R_{2}+R_{3}$ with similar pattern of dark and pale spots. 
Male. Similar to female, except for the following sexual differences. Maxillary palpus length 2.80-3.0 mm $($ mean $=2.90 \mathrm{~mm})$; ratio of length of palpomeres 1-5 to maxillary palpus length, $1=0.042-0.046,2=0.26-0.30$, $3=0.36-0.38,4=0.12-0.15,5=0.15-0.18$, palpomere $20.70-0.80$ (mean $=0.77)$ length of palpomere 3 , palpomere $40.70-0.90($ mean $=0.80)$ length of palpomere 5, maxillary palpus length $1.50-1.55$ (mean $=1.53$ ) forefemur length, maxillary palpus length 0.95 $1.05($ mean $=1.00)$ proboscis length; palpomere 1 with erect dark scales, palpomere 2 with semi-erect dark scales at basal 0.3 , distal 0.7 with decumbent dark scales, a faint dorsal line of pale scales, apex with band of white scales, palpomere 3 dark scaled, with white band at base, with midlongitudinal dorsal line of white scales and white patch at apex, inner surface with tuft of dark brown setae at apex, palpomere 4 dark scaled, dorsally with pale spots at apex and base, inner surface bare, long dark and pale setae at dorsal and ventral margins, palpomere 5 dark scaled, dorsal surface covered with white scales from base to apex, dark and pale setae at outer surface. Antennal length 2.05-2.20 $\mathrm{mm}$ (mean $=2.14 \mathrm{~mm}$ ), pedicel bare with diameter approximately 1.5 of female pedicel; flagellum integument whitish, whorls brown, tufts of long dark setae progressively shorter toward distal segments, flagellomere 1 with tuft of long, narrow white scales dorsally, flagellomeres 13 and 14 integument brown, covered with minute whitish setae. Segment VIII: tergum mostly covered with golden yellow, white and dark scales laterally; sternum dark scaled. Legs: hindtarsomere 2 dark at basal 0.40-0.60 $($ mean $=0.50)$. Genitalia (Figs 1A-D): Ninth tergum not developed, membranous; sternum rectangular, about 0.20 length of gonocoxite laterally. Gonocoxite (Fig. 1A): length about 3.20 maximum width; with five tergomedial setae, a long, slender apicomedial seta. Parabasal seta apically hooked, moderately short, about 3.20 length of its tubercle. Accessory setae hooked at apex, ventral seta about 0.80 length of dorsal seta, about 0.30 length of gonocoxite, dorsal seta about 0.40 length of gonocoxite. Internal seta slender, about 1.05 length of accessory ventral, 0.80 length of accessory dorsal setae. Dorsomedial rim about 0.15 length of gonocoxite. Gonostylus (Fig. 1A): about 0.85 length of gonocoxite, gonostylar claw about 0.45 length of setae $b$, stout, moderately sclerotized. Dorsal claspette (Fig. 1A): about 0.45 length of gonocoxite; apex with 3 setae; setae about 0.40 length of dorsal claspette, curved mesad, dorsal seta with prominent basomesal projection. Ventral claspette (Fig. 1C): about 0.35 length of gonocoxite, without spicules. In ventral aspect, apex wide, laterally produced into two well developed, striated, rounded anterolateral lobes; apically with deep, broad, sclerotized median sulcus between lobes. Preapical plate absent. Basal lobules expanded; refringent structure absent. Phallosome (Fig. 1B): about 0.50 length of gonocoxite and 1.40 length of ventral claspette; aedeagus long, narrow, moderately sclerotized, with hyaline apex, 1.40 wider than long, ventromesal subtriangular projections absent, subapical leaflets moderately sclerotized, slightly serrate, about 0.35 length of phallosome. Proctiger (Fig. 1D): apex hyaline, with wrinkles and sclerotized bars laterally.
Fourth instar larva (Fig. 2A-D). Position and development of setae as figured; range and modal number of branches in Table I. Head (Fig. 2A). Length $0.70-0.80 \mathrm{~mm}$ $($ mean $=0.75 \mathrm{~mm})(\mathrm{n}=4)$, width $0.65-0.75 \mathrm{~mm}($ mean $=$ $0.70 \mathrm{~mm})(\mathrm{n}=4)$, length about 1.10 of width, somewhat ovoid in shape; integument moderately pigmented, yellowish, with dark brown spots and bands (Fig. 2A); collar strongly pigmented dark brown, dorsomentum and ventromentum strongly sclerotized; antenna length 0.35 $0.45 \mathrm{~mm}$ (mean $=0.40 \mathrm{~mm}$ ), width $0.05-0.06 \mathrm{~mm}$ (mean $=0.06 \mathrm{~mm}), 6.00-7.80($ mean $=7.00)$ longer than wide; seta 1 -A inserted $0.10-0.15 \mathrm{~mm}($ mean $=0.12 \mathrm{~mm})$ distant from base of antenna, ratio of distance 1-A to base/ antenna length $0.25-0.35$ (mean $=0.30$ ), ratio of distance 1-A to base/antenna width $1.75-2.35$ (mean $=$ 2,05), 4-A 1.20-1.55 (mean = 1.40) length of 2-A, 4-A 1.40-2.10 (mean $=1.80)$ length of 3-A; distance between bases of 1-C 0.06-0.09 $\mathrm{mm}($ mean $=0.07 \mathrm{~mm})$, length of 2-C $0.25-0.35 \mathrm{~mm}($ mean $=0.30 \mathrm{~mm})$, distance between bases of 2-C 0.03-0.04 $\mathrm{mm}($ mean $=0.04 \mathrm{~mm})$, width of 2-C at base $0.006-0.007 \mathrm{~mm}$ (mean $=0.006 \mathrm{~mm}$ ), 2-C 1.15-1.70 $($ mean $=1.40)$ length of 3-C, 3-C dendritic, strongly sclerotized, length $0.20-0.26 \mathrm{~mm}$ (mean $=0.24 \mathrm{~mm})$, clypeal index 1.35-2.00 $($ mean $=1.60)$, length of 4-C 0.07-0.12 $\mathrm{mm}(\mathrm{mean}=0,09 \mathrm{~mm})$, distance between bases of 4-C and 2-C 0.09-0.13 mm (mean $=0.12 \mathrm{~mm}$ ), distance between bases of 4-C and 3-C 0.08$0.13 \mathrm{~mm}($ mean $=0.11 \mathrm{~mm})$. Thorax (Fig. $2 \mathrm{~B})$ : pro-, meso- and metathorax with continuous midlongitudinal pale stripe, pale yellow to cream; laterad to this, a green, a pale brown and a dark brown longitudinal stripe; setae 1-P multi-branched, with secondary filiform branches, 11, 12, 14-P acicular and long; 2-M forked, 14-M asymmetric and long; 1-T forked, usually double, 9-T acicular. Abdomen (Figs 2B, D): abdominal segments also with midlongitudinal pale stripe, segments I-VII with four tergal plates: a large anteromesal, dark brown, progressively pale brown posteriorly, a small, dark brown posterior plate, and two anterior, very small, rounded, dark brown plates; segment VIII somewhat green in color, with a single anteromesal, dark brown plate, progressively pale brown posteriorly, tergal plate of segment VIII 1.40-1.85 (mean $=1.65$ ) length (from anterior to posterior margin) of tergal plate of segment VII; seta 1-I filiform, symmetric, seta 1-II-VII palmate, leaflets lanceolate, with smooth margins and an irregular, truncate apex. Spiracular apparatus (Fig. 2D): pecten with a dorsoventral dark pigmented line, with $18-23($ mode $=19)$ alternate, long and short teeth, 3-5 long $(\operatorname{mode}=4)$ and 15-19 $($ mode $=$ $15)$ short spines, long spines 2.0-2.80 $($ mean $=2.30)$ length of short spines.

Pupa (Fig. 3A-B): position and development of setae as figured; range and modal number of branches in Table II. Integument lightly tanned, light yellow. Cephalotho$\operatorname{rax}$ (Fig. 3A): head shield and wing, mouthparts and antennal cases weakly pigmented, light yellow; leg cases moderately pigmented; antennal cases with dark areas at flagellomere joints; posterolateral region, above wing cases and lateral portion of metanotum, lateral to setae12-CT, with dark yellow areas; metanotum moderately pigmented medially; $12-\mathrm{CT} 1.15-1.60($ mean $=1.35)$ 


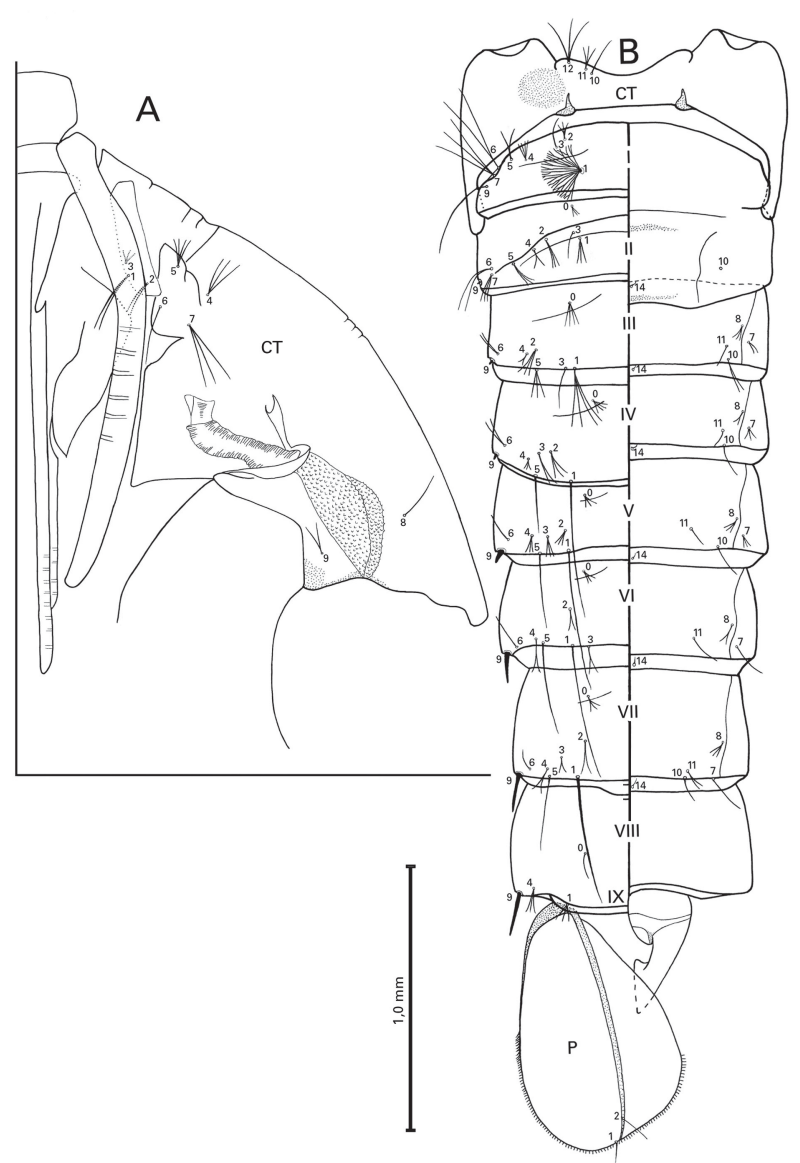

Fig. 3: An. lanei, pupa. A: cephalothorax. B: metathorax (CT); abdominal segments I-IX - left side dorsal; right side ventral; paddle $(\mathrm{P})$. Scales are in $\mathrm{mm}$.

length of 10-CT and 1.35-1.95 (mean $=1.70)$ length of 11-CT, 11-CT 0.70-0.95 $($ mean $=0.80)$ length of 10-CT. Trumpet angusticorn, pinna moderately pigmented, yellowish to light brown, 3.10-4.70 (mean = 3.90) length of meatus. Abdomen (Fig. 3B): abdominal terga and lateral margins of segments I-VIII weakly pigmented, pale yellow; abdominal sterna usually weakly pigmented; anteromesal region of segments II and III moderately pigmented; abdomen length (including segment IX) 2.75$3.75 \mathrm{~mm}($ mean $=3.30 \mathrm{~mm})$; ratio of length of seta 9III/9-II 1.30, 9-IV/9-III 2.40, 9- V/9-IV 2.20, 9-VI/9-V 1.90, 9-VII/9-VI 1.40 and 9-VIII/9-VII 1.05; ratio of length of 9-II/II $0.035,9-$ III/III $0.04,9-I V / I V ~ 0.10,9-\mathrm{V} /$ V 0.20, 9-VI/VI 0.5, 9-VII/VII 0.40 and 9-VIII/VIII 0.40; ratio of length of segment VII/VI 1.01-1.15 (mean = 1.05), VIII/VI 0.90-1.05 (mean $=0.95)$, VIII/VII 0.80$0.95($ mean $=0.90)$; width/length ratio (width at posterior margin) of segment VI 2.05-2.30 (mean = 2.20), VII 1.85-2.10 $($ mean $=1.95)$, VIII 1.90-2.20 $($ mean $=$ 2.05); seta 7-II/6-II ratio about 0.55 . Genital lobe (Fig. 3B): lightly tanned in female, slightly darker in male, male genital lobe approximately 1.70 length of female genital lobe. Paddle (Fig. 3B): weakly pigmented, pale yellow, base of paddle, external buttress and midrib darker than rest of paddle, presence of serration exter- nally at midlateral margin, length of marginal spicules $0.02-0.03 \mathrm{~mm}($ mean $=0.025 \mathrm{~mm})$, paddle length 0.90 $1.05 \mathrm{~mm}($ mean $=0.95 \mathrm{~mm})$, width $0.60-0.75 \mathrm{~mm}$ (mean $=0.65 \mathrm{~mm})$, width/length ratio 0.65-0.75 $($ mean $=0.70)$.

Material examined - Type specimens: paratypes. BRAZIL: SÃO PAULO, Serra da Mantiqueira, Campos do Jordão, Vila Capivari, A.D.F do Amaral, coll.; III-1938, A.L.A Galvão and A.D.F do Amaral det. IV-1938. Deposited in FSP-USP, São Paulo (ACC Nº E-1894, E-1895, E-1896). Not type specimens: 31 specimens were examined, as follows. Brazil, state of São Paulo, CJ and Pindamonhangaba municipalities, Pico do Itapeva $\left(22^{\circ}\right.$ 45.50' S 45 30.87' W), Sallum and Wilkerson coll.; 20XI-2001, Sallum det.: E-12344 F LePe, E-12345 F LePe, E-12346 F LePe, E-12347 F, E-12348 F, E-12349 F LePe, E-12350 M LePe G, E-12351 M LePe G, E-12352 M LePe G, E-12353 F LePe, E-13354 F LePe, E-12355 F LePe, E-12356 F, E-12357 M Pe, E-12358 F LePe, E-12359 F, E-12360 M LePe G, E-12361 F LePe G, E$12362 \mathrm{M}$ LePe, E-12363 M LePe, E-12364 LePe, E12366 LePe, E-12368 Pe, E-12441 LePe, E-12444 Le, E-12445 Le; Sallum et al. coll., 17-II-2006, Senise det.: E- 12917 L, E-12918 L, E-12919 L, E-12920 L. CJ, Barreto et al. coll., XI-1938; Barreto det.: acc. no. 5859 G.

Distribution - An. lanei is probable an endemic species of SM, southeast Brazil. SM is inserted in an endemism center, inside the limits of the biodiversity corridor of Serra do Mar (Aguiar et al. 2003). An. lanei is known from the type locality, CJ and Pico do Itapeva, Pindamonhangaba municipality, both situated in SM. Additionally, this species was reported in San Pedro, Argentina, (Del Ponte 1941) and in the states of Bahia (Figueiredo 1940), Rio de Janeiro (RJ) (Ferreira 1964) and Espírito Santo (Andrade \& Brandão 1957). Del Ponte (1941) captured one adult that was identified as An. lanei, in San Pedro municipality, Argentina. Since then, this species has not been reported in Argentina. Ecological differences among the type locality of An. lanei and the localities in the states of Bahia and Espírito Santo, from where An. lanei was reported, suggest that the specimens may belong to another Nyssorhynchus species. Ferreira (1964) reported An. lanei in RJ; however, there were no details about where the specimens were collected.

Bionomics - Immatures of An. lanei were taken from permanent fresh water habitats, springs and lagoons and also in ditches and ponds connected to streams and rivers (Galvão \& Amaral 1938, Pinto 1939, Galvão 1940, Unti 1943). The water is cold, about $15^{\circ} \mathrm{C}$, and well oxygenated, with a small amount of decomposing organic matter and emergent aquatic vegetation, in the shade, partial or full sun. The species seems to be restricted to high altitudes (Unti \& Ramos 1942, Unti 1943, Ramos 1943). Female adults are zoophilic and exophilic (Galvão \& Amaral 1938, Russel et al. 1943, Unti 1943). In a field collection carried out in Pico do Itapeva, at approximately $1,800 \mathrm{~m}$ above sea level, immatures of $\mathrm{An}$. lanei were found in a ground pool among emergent vegetation. The pool was connected to a fast running, fresh water stream. Anopheles lanei was the only Culicidae found in that larval habitat. 
Results of an infectivity experiment with Plasmodium sp. employing two specimens of An. lanei, showed that neither was infected (Fonseca \& Unti 1943). However, considering the small sample size, it is more plausible to consider that medical importance of the species is unknown.

\section{DISCUSSION}

The subgenus Nyssorhynchus includes three sections, Albimanus, Argyritarsis and Myzorhynchella. The Argyritarsis section is divided into the Albitarsis and Argyritarsis Groups. The latter can be distinguished from the former by the absence of a pair of submedial stripes of white scales on sternum I (Linthicum 1988). The Argyritarsis group is subdivided into four subgroups: Argyritarsis composed of Anopheles argyritarsis Robineau-Desvoidy; Anopheles sawyeri Causey, Deane, Deane and Sampaio; and three monotypic subgroups, i.e., Pictipennis, Darlingi and Lanei. The adult female of Argyritarsis, Darlingi and Lanei can be separated from those of the Pictipennis subgroup by having palpomere 5 entirely pale scaled; flagellomere 1 with pale scales; scutal scales small; foretarsomere 1 without basal pale spot, hindtarsomere 2 dark scaled in less than basal 0.65; wing costal vein with presector pale spot present, and posterolateral scale tufts present on abdominal terga IIIVII. The subgroups Argyritarsis and Lanei can be distinguished from Darlingi by the prehumeral dark spot of the costal vein not greatly enlarged, at most equal to humeral pale spot; palpomere 4 without a patch of white scales, upper mesepimeral scales present and anterior mesepimeral scales absent (Linthicum 1988).

According to Linthicum (1988), female adults of subgroup Lanei can be distinguished from those of subgroup Argyritarsis by possessing hindtarsomere 2 dark scaled on basal 0.55 , humeral and presector pale spots fused, more than 10.0 length of prehumeral dark spot, posterolateral scales tuft present on terga II-VII. However, it is noteworthy that the specimens Linthicum (1988) used to redescribe and illustrate An. lanei may belong to another species of Nyssorhynchus, for reasons which we will explain in the following paragraphs.

When using Linthicum's (1988) adult identification key, it is not possible to key out either specimen of $A n$. lanei collected in the type locality or the type specimens deposited in the FSP-USP collection. The diagnostic characters of Linthicum, contrast with those of Galvão and Amaral (1938) and Forattini (1962) because in An. lanei, the posterolateral scales tuft is always absent on tergum II. However, Linthicum used the presence of posterolateral scales tuft on tergum II to separate An. lanei from An. sawyeri and An. argyritarsis. Within the Argyritarsis section only Anopheles braziliensis (Chagas) and Anopheles darlingi Root possess posterolateral scale tufts on tergum II. Moreover, Linthicum's specimen differs from An. braziliensis by the absence of submedial stripes of white scales on sternum I, and from $A n$. darlingi by not possessing prehumeral dark spot greatly enlarged and anterior mesepimeral scales always absent. More importantly, male genitalia characteristics of the specimen illustrated by Linthicum (1988) are distinct from those of Galvão and Amaral (1938). Also, when using Forattini's (1962) male genitalia identification key, Linthicum's specimen was keyed out as a species that is morphologically similar to An. argyritarsis, whereas it is impossible to identify the adult as any species of the Argyritarsis section. In examining the material examined by Linthicum, we noted that three paratypes, one female adult and two male genitalia, were the only specimens examined, and all of them were collected in CJ. However, the illustration on page 261 shows the male genitalia of a specimen collected in ET. This specimen is not included in Linthicum's list. Consequently, we believe that the illustration was incorrectly labeled as An. lanei. Additionally, ecological differences between ET and CJ area support the hypothesis that the male genitalia of the specimen from ET may belong to a species of the Argyritarsis section that is morphologically similar to An. argyritarsis, perhaps an undescribed species of this section, while the adult female may belong to a species of the Argyritarsis section, which was mislabeled as An. lanei. The region of $\mathrm{CJ}$ is situated in SM, 1,500 $\mathrm{m}$ above sea level with a low annual mean temperature, below $15^{\circ} \mathrm{C}$, while ET is situated in a restinga vegetation area, near the coast, $30 \mathrm{~m}$ above sea level and annual mean temperature above $18^{\circ} \mathrm{C}$ (Müller 1974, Nimer 1989). Finally, comparing specimens, which were recently collected in Pico do Itapeva, Pindamonhangaba, CJ area, with the type specimens, it is possible to identify them with confidence as An. lanei of Galvão and Amaral. These specimens include male and female adults with associated immatures.

An. lanei can be separated from An. darlingi, An. argyritarsis and An. sawyeri by a small prehumeral dark and a minute humeral dark spots on costal vein, which can be sometimes absent; in the male genitalia, by having the aedeagus with a hyaline apex, wider than long, slightly serrate subapical leaflets, and by the characteristic shape of ventral claspette. Additionally, An. lanei can be separated from An. darlingi by the absence of anterior mesepimeral pale scales and posterolateral tuft of scales absent on tergum II; from An. argyritarsis by the presence of a distinct pale ring at apex of hindtarsomere 1, whereas it is usually absent in the latter and, when present, is very small; abdominal terga scales mostly white, while in An. argyritarsis scales are predominantly yellow; in the male genitalia, by the presence of basomesal projections on setae of dorsal claspette. An. lanei can be separated from An. sawyeri by the presence of apical pale rings in midtarsomeres 1 and 2, and absence of a triangular dark spot on prescutellar area; ratio of palpus length and proboscis length of An. lanei is approximately 1, while in An. sawyeri it is about 0.85; apex of the aedeagus of An. lanei is hyaline and conical, without ventromesal subtriangular projection, whereas in An. sawyeri the apex of the aedeagus is somewhat round, as wide as long, and ventromesal subtriangular projection is present.

Because there were no immature specimens of $A n$. lanei available to Linthicum (1988), the diagnostic characteristics of the fourth instar larva and pupa refer to those of Galvão and Amaral (1938), Galvão $(1940,1943)$ and Lane (1953). Consequently, the fourth instar larva 
identification key allows a correct identification of specimens of An. lanei. Similar to other members of Nyssorhynchus, fourth instar larva of An. lanei (Fig. 2AD) have abdominal setae 1-III-VII palmate, with smoothmargined lanceolate leaflets, and setae 5-7-C plumose. Generally, it is not possible to separate both Argyritarsis and Albimanus sections by the fourth instar larval characteristics as can be observed in Faran and Linthicum's (1981) and Linthicum's (1988) identification keys. Comparing fourth instar larva of An. lanei with those of other species of the Argyritarsis section, it is evident that it is morphologically similar to An. argyritarsis, An. sawyeri and $A n$. darlingi by possessing a non-palmate seta 1-P; however, it can be distinguished from these species by having a small clypeal index and seta 3-C strongly sclerotized and dendritic. Additionally, fourth instar larva of $A n$. lanei can be easily distinguished from that of $A n$. darlingi in having seta $13-\mathrm{S}$ poorly developed.

In the pupal stage, An. lanei (Figs 3A-B) is similar to An. argyritarsis, An. sawyeri, An. pictipennis Philippi, and An. darlingi of the Argyritarsis group. It separates from An. darlingi by having an angusticorn trumpet, whereas in An. darlingi the trumpet is laticorn and from An. argyritarsis and An. sawyeri by having seta 3-CT (Fig. 3A) shorter than that in the other two species. To confirm the importance of seta 3-CT to separate $A n$. lanei from An. argyritarsis and An. sawyeri, a careful evaluation of this character will be necessary.

In order to use Linthicum's (1988) identification key, we propose some modifications that will make identification of specimens of An. lanei possible.

\section{MALE GENITALIA}

2 (1). Apex of aedeagus longer than wide, ventromesal subtriangular projections encircling entire subapical region forming a distinct collar; ventral claspette with apex moderately wide and truncate, median sulcus small, occasionally indistinct; dorsal seta of dorsal claspette with a well-developed basomesal projection (Darlingi subgroup). .5. An. darlingi

Apex of aedeagus wider than long, ventromesal subtriangular projections absent or, if present, never forming a collar; ventral claspette never truncate, median sulcus well developed; dorsal seta of dorsal claspette with or without a basomesal projection.

3 (2). Ventral claspette with apex expanded laterally into large rounded lobes directed caudally; distal third constricted, forming a distinct waist; aedeagus without ventromesal subtriangular projections, subapical leaflets slightly serrate and relatively long; apex of the dorsal claspette with 3 setae, dorsal seta with prominent basomesal projection (Lanei subgroup)........3. An. lanei

Ventral claspette with apex not expanded laterally into large rounded lobes, distal third not constricted at middle region, not forming a waist, somewhat straight; aedeagus with ventromesal subtriangular projections, subapical leaflets strongly serrate; apex of the dorsal claspette with 2 or 3 setae, dorsal seta with basomesal projection absent, when present, not prominent (Argyritarsis subgroup).

\section{FEMALE}

4 (3). Minute sub-basal dark spot either present or absent, when absent, humeral and sub-basal pale spots fused; terga II-VI with pale scales and bilateral spots of dark scales posteriorly; hindtarsomere 1 with a distinct pale ring at apex (Lanei subgroup).............. An. Aanei

Distinct sub-basal dark spot, always present; terga IIVI either with purple scales medially and laterally with yellow scales or medially with cream scales and some brown scales on apical 0.5 of segment; hindtarsomere dark scaled at apex, with few pale scales at joint (Argyritarsis sub-

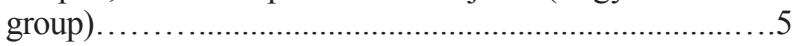

\section{LARVA}

1. Setae 3-C strongly dendritic (Argyritarsis Group, Lanei subgroup).........................................An. lanei

Setae 3-C never dendritic.........................................2

2. Seta 1-P plumose or fanlike with thin branches, never palmate, with lanceolate branches; 1-2-P never inserted on a common sclerotized tubercle; 3-T, 1-T fanlike with thin branches or weakly palmate with narrow lanceolate branches or palmate with well- developed lanceolate branches, if palmate with well-developed lanceolate branches then 13-S very strongly developed, 2.2-2.5 length of saddle (Argyritarsis group, Argyritarsis subgroup)........3

Seta 1-P always palmate with lanceolate branches; 1,2-P inserted on a common sclerotized tubercle; 3-T, 1-I always palmate, with well-developed lanceolate branches (Albitarsis group).................................................

\section{ACKNOWLEDGEMENTS}

To Henry R. Rupp for kindly reviewing the English.

\section{REFERENCES}

Aguiar AP, Chiarello AG, Mendes SL, de Matos EN 2003. The Central and Serra do Mar corridors in the Brazilian Atlantic forest. In C Galindo-Leal, IG Câmara (eds), The Atlantic forest of South America: biodiversity status, threats and outlook, Island Press, Washington, p. 118-132.

Andrade RM de, Brandão H 1957. Contribuição para o conhecimento da fauna de anofelinos do Estado do Espírito Santo. Área de distribuição e incidência de espécies por cidades, vilas e povoados (Diptera, Culicidae). Rev Bras Malariol Doencas Trop 9: 391-416.

Belkin JN 1962. The mosquitoes of South Pacific (Diptera: Culicidae), University of California Press, Berkley and Los Angeles, 608 pp.

Del Ponte E 1941. Revision de las espécies argentinas del genero Anopheles, Faculdad de Ciencias Medicas, Universidad de Buenos Aires, Buenos Aires, 113 pp.

Faran ME 1980. Mosquito Studies (Diptera: Culicidae) XXXIV -A revision of the Albimanus section of the subgenus Nyssorhynchus of Anopheles. Contrib Amer Ent Inst 15: 1-215.

Faran ME, Linthicum KJ 1981. A handbook of the Amazonian species of Anopheles (Nyssorhynchus) (Diptera: Culicidae). Mosq Syst 13: 1-81.

Ferreira E 1964. Distribuição geográfica dos anofelíneos no Brasil e sua relação com o estado atual da erradicação da malária. Rev Bras Malariol Doencas Trop 16: 329-348.

Figueiredo B 1940. Uma inspeção de malária em Jitaúna. In 
Sociedade de Medicina da Bahia, Anais da Sociedade de Medicina da Bahia - Jornadas Médicas, Imprensa Vitória, Bahia, p. 110-129.

Fonseca JAB, Unti O 1943. Infecção experimental de anofelinos de regiões indenes à malária. Folia Clin Biol (São Paulo) 15: 43-52.

Forattini OP 1962. Entomologia Médica: Parte geral, Diptera, Anophelini, Faculdade de Higiene e Saúde Pública, Departamento de Parasitologia, São Paulo, 662 pp.

Forattini OP 1996. Culicidologia Médica, Universidade de São Paulo, São Paulo, 548 pp.

Forattini OP 2002. Culicidologia Médica, Universidade de São Paulo, São Paulo, 860 pp.

Galvão ALA 1940. Contribuição ao conhecimento dos anofelinos do grupo Nyssorhynchus de São Paulo e regiões vizinhas (Diptera: Culicidae). Arq Zool S Paulo 1: 399-484.

Galvão ALA 1943. Chaves para determinação das espécies do subgênero Nyssorhynchus do Brasil. Arq Hig Saude Publica 8: 141-162.

Galvão ALA, do Amaral ADF 1938. Sobre um novo anofelino de Campos do Jordão, Estado de São Paulo, Anopheles (Nyssorhynchus) lanei n.sp. (Diptera, Culicidae). Rev Biol Hyg 9: 8-16.

Harbach RE 2004. The classification of genus Anopheles (Diptera: Culicidae): A working hypothesis of phylogenetic relationships. Bull Entomol Res 94: 537-553.

Harbach RE, Knight KL 1980. Taxonomist's glossary of mosquito anatomy, Plexus Publishing, Marlton, New Jersey, 415 pp.

Lane J 1939. Catálogo dos mosquitos Neotropicos. Boletim Biológico. Série Monográfica n. 1, Clube Zoológico do Brasil, São Paulo, 218 pp.
Lane J 1953. Neotropical Culicidae, Universidade de São Paulo, São Paulo, 548 pp.

Levi-Castillo R 1949. Atlas de los anofelinos Sudamericanos, Tipografia de la Sociedad Filantrópica del Guayas, Guayaquil, $206 \mathrm{pp}$.

Linthicum KJ 1988. A revision of the Argyritarsis section of the subgenus Nyssorhynchus of Anopheles. Mosq Syst 20: 98-271.

Müller P 1974. Aspects of Zoogeography, Dr. W Junk BV Publishers, The Hague, 208 pp.

Nimer E 1989. Climatologia do Brasil, IBGE - Fundação Instituto Brasileiro de Geografia e Estatística, Departamento de Recursos Naturais e Estudos Ambientais, Rio de Janeiro, 421 pp.

Pinto C 1939. Disseminação da malaria pela aviação: Biologia do Anopheles gambiae e outros anofelíneos do Brasil. Mem Inst Oswaldo Cruz 34: 293-430.

Ramos AS 1943. Observações sobre os anofelinos do litoral Paulista. Arq Hig Saude Publica 8: 23-31.

Russel PF, Rozeboom LE, Stone A 1943. Keys to the Anopheline mosquitoes of the world: with notes on their identification, distribution and relation to malaria, The American Entomological Society, The Academy of Natural Sciences, Philadelphia, $152 \mathrm{pp}$.

Unti O 1943. Oxigênio dos focos de anofeles de São Paulo. Arq Hig Saude Publica 8: 85-102.

Unti O, Ramos AS 1942. Anofelismo das alturas no Brasil meridional. Arq Hig Saude Publica 7: 91-106.

Wilkerson RC, Peyton EL 1990. Standardized nomenclature for the costal wing spots of the genus Anopheles and other spotted-wing mosquitoes (Diptera: Culicidae). J Med Entomol 27: 207-224. 
